\title{
Suppression of Mutant Huntingtin Aggregate Formation by Cdk5/p35 through the Effect on Microtubule Stability
}

\author{
Sayuko Kaminosono, ${ }^{1 *}$ Taro Saito, ${ }^{1 *}$ Fumitaka Oyama, ${ }^{2}$ Toshio Ohshima, ${ }^{3}$ Akiko Asada, ${ }^{1}$ Yoshitaka Nagai, ${ }^{4}$ \\ Nobuyuki Nukina, ${ }^{2}$ and Shin-ichi Hisanaga ${ }^{1}$ \\ ${ }^{1}$ Department of Biological Sciences, Tokyo Metropolitan University, Hachioji, Tokyo 192-0397, Japan, ${ }^{2}$ Laboratory for Structural Neuropathology, RIKEN \\ Brain Science Institute, Wako, Saitama 351-0198, Japan, ${ }^{3}$ Department of Life Science and Medical Bio-Science, Waseda University, Tokyo 162-8480, Japan, \\ and ${ }^{4}$ Division of Clinical Genetics, Department of Medical Genetics, Osaka University Graduate School of Medicine, Suita, Osaka 565-0871, Japan
}

Huntington's disease $(\mathrm{HD})$ is a polyglutamine $[\mathrm{poly}(\mathrm{Q})]$ disease with an expanded poly $(\mathrm{Q})$ stretch in the $\mathrm{N}$ terminus of the huntingtin protein (htt). A major pathological feature of HD neurons is inclusion bodies, detergent-insoluble aggregates composed of poly $(\mathrm{Q})$ expanded mutant htt (mhtt). Misfolding of mhtt is thought to confer a toxic property via formation of aggregates. Although toxic molecular species are still debated, it is important to clarify the aggregation mechanism to understand the pathogenesis of mhtt. We show $\mathrm{Cdk} / \mathrm{p} 35$ suppresses the formation of mhtt inclusion bodies in cell lines and primary neurons. Although we expressed the N-terminal exon 1 fragment of htt lacking phosphorylation sites for Cdk5 in COS-7 cells, the kinase activity of Cdk5 was required for the suppression. Furthermore, Cdk5/p35 suppressed inclusion formation of atrophin-1, another poly $(\mathrm{Q})$ protein, raising the possibility that Cdk5/p35 generally suppresses inclusion formation of poly $(\mathrm{Q})$ proteins. Microtubules (MTs) were a downstream component of Cdk5/p35 in the suppression of inclusion formation; $\mathrm{Cdk} 5 / \mathrm{p} 35$ disrupted MTs, which were required for the formation of inclusions. Moreover, stabilization of MTs by Taxol induced inclusions even with overexpression of Cdk5/p35. The formation of inclusions was also regulated by manipulating the Cdk5/p35 activity in primary rat or mouse cortical neuron cultures. These results indicate that Cdk5-dependent regulation of MT organization is involved in the development of aggregate formation and subsequent pathogenesis of poly $(\mathrm{Q})$ diseases. This Cdk5 inhibition of htt aggregates is a novel mechanism different from htt phosphorylation and interaction with Cdk5 reported previously (Luo et al., 2005; Anne et al., 2007).

Key words: Huntington's disease; huntingtin; polyglutamine; Cdk5/p35; microtubule; aggregate formation

\section{Introduction}

Huntington's disease $(\mathrm{HD})$ is a polyglutamine $[$ poly $(\mathrm{Q})]$ disease with an expanded poly $(\mathrm{Q})$ tract in the huntingtin (htt) protein. $\mathrm{HD}$ is characterized by involuntary movements, personality changes, and dementia, caused mainly by selective loss of medium-sized spiny neurons in the striatum. One of the major pathological features found in HD neurons is the presence of detergent-insoluble ubiquitinated inclusions composed of poly (Q)-expanded mutant htt (mhtt) (Davies et al., 1997; Scherzinger et al., 1997). However, the manner by which mhtt causes neurodegeneration is not well understood. Currently, no treatment exists to prevent or delay the appearance and progression of symptoms or death of HD patients.

$\mathrm{htt}$ is a large protein with a molecular mass of $350 \mathrm{kDa}$ lacking

Received Nov. 14, 2007; revised June 28, 2008; accepted July 21, 2008.

This work was supported in part by Grants-in-Aid for Scientific Research on Priority Area (Research on Pathomechanisms of Brain Disorders) from Ministry of Education, Culture, Sports, Science, and Technology of Japan (S.-i.H.). We thank Dr. Mitsuo Tagaya (Tokyo University of Pharmacy and Life Science, Tokyo, Japan) for providing FLAG-dynamitin.

*S.K. and T.S. contributed equally to this work.

Correspondence should be addressed to Shin-ichi Hisanaga, Department of Biological Sciences, Graduate School of Science, Tokyo Metropolitan University, 1-1 Minami-osawa, Hachiohji, Tokyo 192-0397, Japan. E-mail: hisanaga-shinichi@tmu.ac.jp.

DOI:10.1523/JNEUROSCI.0973-08.2008

Copyright $\odot 2008$ Society for Neuroscience $\quad$ 0270-6474/08/288747-09\$15.00/0 similarity to other proteins. htt protein has a poly $(\mathrm{Q})$ stretch in the middle of exon 1. In normal individuals, the length of the $\operatorname{poly}(\mathrm{Q})$ tract is $<35$ repeats, but with expansion beyond 40 , the htt protein is misfolded, becomes toxic, forms aggregates, and causes disease. Formation of large aggregates is a multiple-step process from a misfolded monomer to large perinuclear or intranuclear deposits called inclusion bodies (Tanaka et al., 2003; Taylor et al., 2003; Nagai et al., 2007). The toxicity of the aggregates in neurons has been attributed to a variety of causes, including a defect in RNA synthesis, cell survival activity, microtubule (MT)-dependent trafficking, or the ubiquitin-proteasome system (DiFiglia et al., 1997; Tobin and Signer, 2000; Harjes and Wanker, 2003; Gauthier et al., 2004; Li and Li, 2004; Cattaneo et al., 2005; Bennett et al., 2007). Inhibiting poly(Q) aggregation alleviates the symptoms of HD patients, as reported in Drosophila and mouse models of HD (Kazantsev et al., 2002; Sánchez et al., 2003). Therefore, it is important not only to delineate the aggregation mechanism of mhtt but also to identify the factors that induce an environment permissive to this neurodegeneration.

Cdk5 is a member of the CDK (cyclin-dependent kinase) family, activated predominantly in postmitotic neurons by an activation subunit, p35 or p39. Although Cdk5 plays an important physiological role in neuronal development and synaptic activity (Dhavan and Tsai, 2001; Cheung and Ip, 2007), its deregulation 
by calpain-mediated cleavage of p35 to p25 is implicated in neuronal cell death of several neurodegenerative diseases such as Alzheimer's and Parkinson's disease (Dhavan and Tsai, 2001; Hisanaga and Saito, 2003; Shelton and Johnson, 2004). It is recently reported that $\mathrm{Cdk} 5$ phosphorylates htt at Ser434 to reduce $\mathrm{N}$-terminal cleavage, resulting in decreased aggregation (Luo et al., 2005), and at Ser1181 and Ser1201 to prevent the gain of toxic activity (Anne et al., 2007).

We studied the role of Cdk5 in mhtt aggregate formation in cell lines and in primary neuronal cultures. On coexpression of $\mathrm{Cdk} 5 / \mathrm{p} 35$ with an N-terminal exon $1 \mathrm{htt}$ fragment (tNhtt) without phosphorylation sites for $\mathrm{Cdk} 5 / \mathrm{p} 35$, the formation of $\mathrm{mhtt}$ inclusions was suppressed. We then explored the mechanism whereby $\mathrm{Cdk} 5 / \mathrm{p} 35$ inhibits the formation of inclusions and found that it acts via MT organization.

\section{Materials and Methods}

Antibodies, plasmids, and chemicals. Sources of reagents are as follows: Cdk5 (DC17) and p35 (C-19) antibodies (Santa Cruz Biotechnology); anti-GFP antibody (Roche Diagnostics); anti- $\beta$-tubulin and anti-actin antibodies (Sigma-Aldrich); roscovitine (Calbiochem); anti-histone H3 antibody (Cell Signaling). Human htt exon 1-enhanced green fluorescent protein (EGFP) coding region was excised from pIND-htt exon 1-EGFP (Wang et al., 1999) by HindIII and NotI digestion and inserted into the corresponding sites of pcDNA3.1 (Invitrogen). pFLAG-dynamitin was provided by Dr. Mitsuo Tagaya (Tokyo University of Pharmacy and Life Science, Tokyo, Japan) (Hirose et al., 2004). Atrophin-1-EGFP with 19Q or 81Q (Yamada et al., 2001), pCMV-dominant-negative Cdk5 (K33T or D144N) (Nikolic et al., 1996; Saito et al., 2003), and pSG5-tau (Sakaue et al., 2005) have been described previously.

Cell culture, transfection, and fluorescence microscopic observation. COS-7 and Neuro2a cells were cultured in DMEM supplemented with $10 \%$ FBS and transfected with tNhtt-poly(Q)-EGFP and/or pCMVCdk5 and pCMV-p35 using PolyFect Transfection Reagent (QIAGEN) according to the manufacturer's protocol. Transfection of pSG5-tau or pFLAG-dynamitin was performed similarly, and the expression was confirmed by immunoblotting.

COS-7 cells were treated with $100 \mathrm{ng} / \mathrm{ml}$ nocodazole to disassemble MTs for $18 \mathrm{~h}$ starting $6 \mathrm{~h}$ after transfection or for $6 \mathrm{~h}$ starting $18 \mathrm{~h}$ after transfection. COS-7 cells were treated with $20 \mu \mathrm{M}$ Taxol to stabilize MTs or with $20 \mu \mathrm{M}$ roscovitine to inhibit Cdk5 activity for $18 \mathrm{~h}$ starting $6 \mathrm{~h}$ after the transfection. After fixation with $4 \%$ paraformaldehyde, aggregate formation was analyzed under an Axioskop microscope (Carl Zeiss) or a LSM5 Pascal confocal laser microscope (Carl Zeiss). Intracellular inclusions were defined as bright EGFP-positive deposits with weak cytoplasmic EGFP fluorescence. EGFP deposits were often observed without cytoplasmic staining, but these deposits were excluded from counting in this study. More than 300 transfected cells were counted in a minimum of five independent transfection experiments. Data were analyzed statistically by one-sided, paired Student's $t$ test and expressed as mean \pm SEM in each figure. The ratio of cells with inclusions was defined as a percentage of the total number of EGFP- or EGFP/Cdk5-positive cells. Cdk5-positive cells were visualized by immunostaining with antiCdk5 (DC17) antibody. Anti- $\beta$-tubulin staining was performed as described previously (Sakaue et al., 2005).

Primary neurons were prepared from 17 -d-old embryonic rat brain cerebral cortex. Adenovirus carrying FLAG-tagged p35 was infected as described previously (Saito et al., 2003). Adenovirus empty vector or mock infection was used as control. Transfection of tNhtt-poly(Q)EGFP was performed by the calcium phosphate method on cultures on the sixth day in vitro (DIV6). Primary neurons were transfected with short interfering RNA (siRNA) and tNhtt-poly(Q)-EGFP at DIV5 or DIV9 using Lipofectamine 2000 reagent (Invitrogen) according to the manufacturer's instructions. SMARTpool siRNA for rat Cdk5 (ONTARGETplus SMARTpool siRNA; Dharmacon L-094624-01-0020) was obtained from GE Healthcare. Neurons were observed 24 or $48 \mathrm{~h}$ after transfection. Neurons were treated with $20 \mu \mathrm{M}$ Taxol to stabilize MTs for
$18 \mathrm{~h}$ starting $6 \mathrm{~h}$ after transfection. After fixation with $4 \%$ paraformaldehyde, aggregate formation was analyzed under an Axioskop microscope.

$\mathrm{Cdk} 5^{-1-}$ mice were generated and maintained as described previously (Ohshima et al., 1996; Yamashita et al., 2007). Mice were handled in accordance with institutional guidelines and housed in a pathogen-free environment on a $12 \mathrm{~h}$ light/dark cycle (Ohshima et al., 2007). Primary neurons were prepared from 16.5 -d-old embryonic Cdk $5^{+/+}, \mathrm{Cdk} 5^{+/-}$, or Cdk5 $5^{-1-}$ mice. Genotypes of the offspring were assessed using PCR as described previously (Ohshima et al., 2007; Yamashita et al., 2007). Cultured neurons were transfected with tNhtt-poly(Q)-EGFP at DIV5 using Lipofectamine 2000 reagent (Invitrogen). Neurons were observed $24 \mathrm{~h}$ after transfection.

Estimation of MT polymers. MT polymers were estimated by the procedures of Nguyen et al. (1999) with some modifications. Briefly, COS-7 cells in $35 \mathrm{~mm}$ dishes, which were pretreated with $100 \mathrm{ng} / \mathrm{ml}$ nocodazole or $20 \mu \mathrm{M}$ Taxol for $18 \mathrm{~h}$, or transfected with Cdk5 and p35, were gently suspended in MT-stabilizing buffer (0.1 м PIPES, pH 6.8, 1 mм EGTA, 1 mм $\mathrm{MgCl}_{2}, 20 \%$ glycerol, $0.5 \%$ Nonidet P- $40,0.1 \mathrm{~mm}$ GTP). After incubation for $10 \mathrm{~min}$ at $37^{\circ} \mathrm{C}$, the suspensions were centrifuged at $1100 \times g$ for $3 \mathrm{~min}$. The supernatant was removed and an identical volume of SDS-PAGE sample buffer was added to the resulting pellets containing MTs. The amounts of tubulin in the supernatant and MT-polymers were detected by immunoblotting after SDS-PAGE with histone H3 as a loading control.

Immunoprecipitation, immunoblotting, and in vitro phosphorylation by $C d k 5 / p 35$. The binding of Cdk5 or p35 with tNhtt-poly(Q)-EGFP was examined by immunoblotting with anti-Cdk 5 or anti-p 35 antibody after immunoprecipitation of tNhtt-poly(Q)-EGFP from COS-7 cells with anti-GFP antibody (Saito et al., 2003). The longer 90Q form was phosphorylated by Cdk5/p25 purified from Sf9 cells in MOPS (3morpholinepropanesulfonic acid) buffer containing $0.5 \%$ Nonidet P-40 and $\left[\gamma_{-}{ }^{32} \mathrm{P}\right]$ ATP $(0.1 \mathrm{~mm})$ for $30 \mathrm{~min}$ at $35^{\circ} \mathrm{C}$ (Sakaue et al., 2005). Phosphorylation was estimated by a FLA7000 Image Analyzer (Fuji Film) after SDS-PAGE.

\section{Results}

\section{Suppression of mhtt aggregate formation by Cdk5/p35}

We used the N-terminal exon 1 fragment of huntingtin ( $\mathrm{tNhtt}$ ) including the $\operatorname{poly}(\mathrm{Q})$ stretch in the middle, which is much shorter than those used previously by Luo et al. (2005) and Anne et al. (2007). The effect of Cdk5/p35 on tNhtt aggregate formation was examined by transient expression in COS-7 cells, frequently used for studies on mhtt aggregation (Onodera et al., 1997; Hazeki et al., 1999). When tNhtt with 16Q, 50Q, or 90Q (tNhtt-16Q, 50Q, or 90Q-EGFP; these constructs will be referred to $16 \mathrm{Q}, 50 \mathrm{Q}$, and 90Q hereafter), 50Q and 90Q displayed bright fluorescent foci (Fig. 1A) as reported previously (Onodera et al., 1997; Hazeki et al., 1999; Wyttenbach et al., 2000), whereas 16Q was distributed diffusely in the cytoplasm. Cotransfection with Cdk5/p35 dramatically reduced the number of cells containing inclusions (Fig. 1A). Triple expression in the same cells is shown in supplemental Figure S1 $A$ (available at www.jneurosci.org as supplemental material). The effect of Cdk5/p35 was also confirmed biochemically by immunoblot analysis. Expression levels of proteins are indicated in supplemental Figure S1 B (available at www.jneurosci.org as supplemental material). The formation of SDS-insoluble aggregates was detected in the stacking gel by immunoblotting when either $50 \mathrm{Q}$ or $90 \mathrm{Q}$ was expressed (Fig. $1 B$; supplemental Fig. S1 B, available at www.jneurosci.org as supplemental material). The SDS-insoluble aggregates disappeared when $50 \mathrm{Q}$ or $90 \mathrm{Q}$ was coexpressed with Cdk5/p35 (Fig. 1B). Even when Cdk5/p35 activity was decreased by reducing the amount of transfected Cdk5 and p35 plasmid DNA, the inclusions were completely abolished (Fig. $1 C$ ). We repeated the same experiments with Neuro2a neuroblastoma cells, to exclude the possibility that the inhibitory effect of $\mathrm{Cdk} 5 / \mathrm{p} 35$ was cell type- 

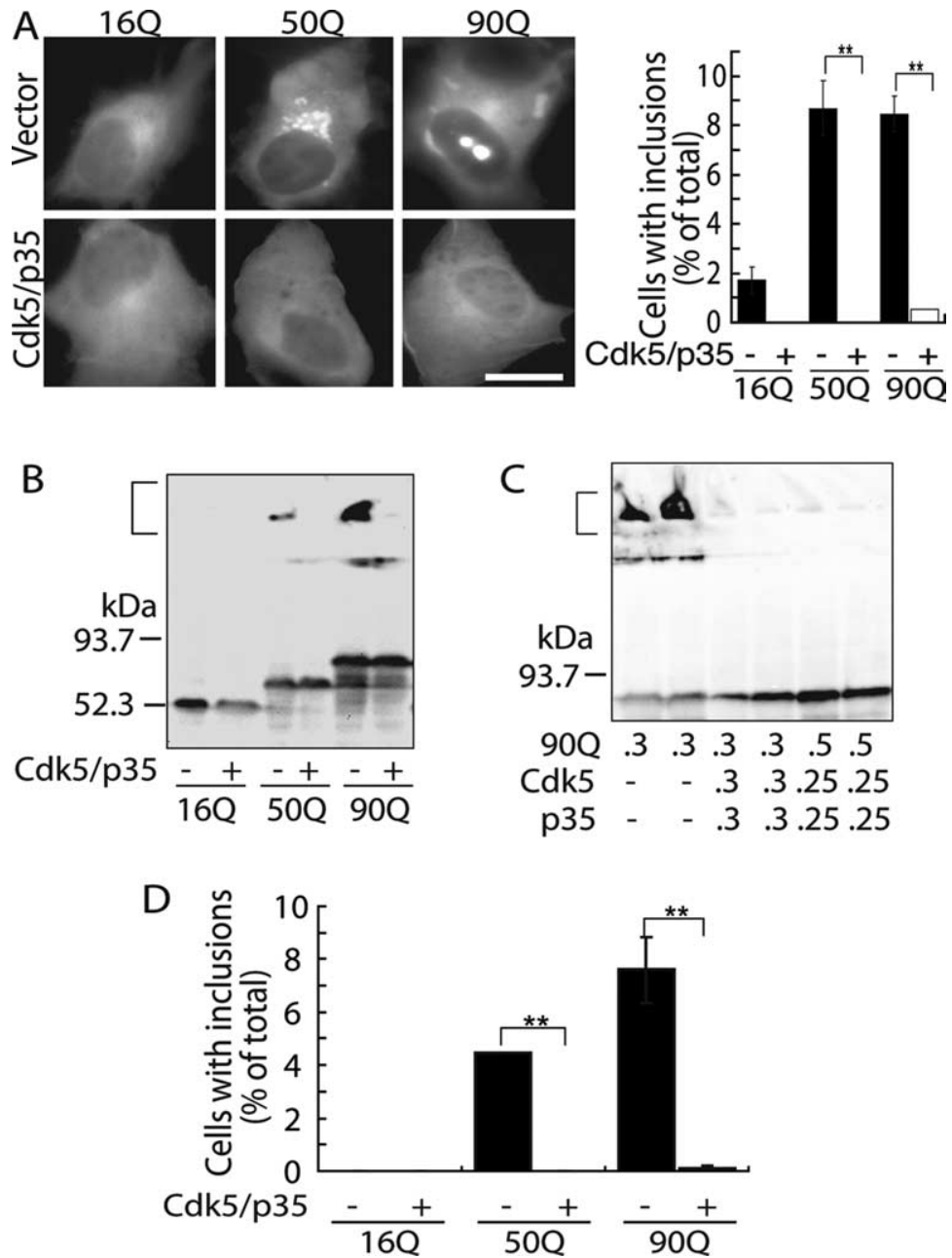

Figure 1. Suppression of the formation of mhtt inclusions by $C \mathrm{dk} 5 / \mathrm{p} 35 . A$, Fluorescence microscopic images of tNhtt-160,500, or 90Q-EGFP expressed alone (top panels) or coexpressed with Cdk5/p35 (bottom panels) in COS-7 cells (left panels). Cells were analyzed for inclusion formation $24 \mathrm{~h}$ after transfection. Scale bar, $10 \mu \mathrm{m}$. Quantification of cells containing EGFP inclusions is shown in the right panel (mean $\pm S E M ;{ }^{* *} p<0.001$ ). $\boldsymbol{B}$, Detection of SDS-insoluble aggregates in COS-7 cells expressing 500 or 900 but not 160 by anti-GFP immunoblotting. A bracket indicates aggregates in the stacking gel. $C$, Inhibition of tNhtt-90Q-EGFP inclusion formation by $\mathrm{Cdk} 5 / \mathrm{p} 35$ when cotransfected in different plasmid ratios. The amount of plasmid DNA (in micrograms) used is indicated below the blot. A bracket indicates SDS-insoluble aggregates. $D$, Inclusion formation of tNhtt-16Q, 50Q, 90Q EGFP and suppression by Cdk5/p35 in Neuro2a cells. The number of cells containing EGFP-aggregates was counted and expressed as the percentage ratio to total EGFP-positive cells transfected in the presence $(+)$ or absence $(-)$ of $\mathrm{Cdk5/p35}$ (mean \pm SEM; $\left.{ }^{* *} p<0.001\right)$.

specific. Cdk5/p35 also suppressed the formation of inclusions in these cells (Fig. 1D). Collectively, these results indicate that Cdk5/p35 suppresses aggregate formation of mhtt in cultured cells.

\section{The kinase activity of Cdk5 is required for suppression of mhtt inclusion body formation}

It was previously reported that C $\mathrm{dk} 5$ physically binds htt (Luo et al., 2005). To examine this molecular interaction, we examined coimmunoprecipitation of mhtt with anti-Cdk5 or anti-p35 antibody, or of Cdk5 or p35 with anti-GFP antibody, but we could not detect binding of either Cdk5 or p35 to any tNhtt-poly(Q)EGFP proteins under our experimental conditions (data not shown). We also assessed the respective effects of Cdk5 and p35 on the appearance of inclusions (Fig. $2 A$ ). Inclusions were slightly reduced by cotransfection with $\mathrm{Cdk} 5$, but this effect was not statistically significant. Cotransfection with p35 was more effective in decreasing inclusions. This may be attributable to the activation of endogenous Cdk5 in COS-7 cells. In contrast, coexpression of both Cdk5 and p35 dramatically reduced inclusions of both 50Q and 90Q (Fig. 2A).

p35 is cleaved to p25 by calpain to generate Cdk5/p25, which has increased kinase activity (Patrick et al., 1999; Hisanaga and Saito, 2003; Sakaue et al., 2005). We observed a similar inhibitory effect of Cdk5/p25 on inclusions (Fig. 2 B). Two activator proteins for Cdk5 are p35 and p39 (Dhavan and Tsai, 2001; Hisanaga and Saito, 2003). Cdk5/p39 potently inhibited inclusion formation, similar to that observed with Cdk5/p35 (Fig. 2B). To confirm the requirement for Cdk5 kinase activity in the inhibition of inclusion body formation, we used the Cdk5 inhibitor roscovitine. Roscovitine abolished the inhibitory activity of Cdk5 for mhtt inclusion formation (data not shown). We confirmed this result with kinase-negative mutants of Cdk5, Cdk5-D144N, and Cdk5-K33T (Nikolic et al., 1996; Saito et al., 2003) (our unpublished results). Statistically significant inhibition was not observed with kinase-negative mutants of Cdk5 (Fig. 2C). These results clearly indicate that the kinase activity of Cdk5 is necessary for the suppression of mhtt inclusion formation.

The above results suggested the possibility that tNhtt-poly(Q)-EGFP is phosphorylated by Cdk5/p35. Although we considered this possibility unlikely because the constructs were short exon 1 fragments with no predicted Cdk5 phosphorylation sites, we evaluated this hypothesis experimentally. 90Q isolated from COS-7 cells by immunoprecipitation was incubated with $\mathrm{Cdk} 5 / \mathrm{p} 25$, but no phosphorylation was observed (data not shown). The fact that Cdk5 neither interacted with nor phosphorylated tNhttpoly(Q)-EGFP implies that the effect of Cdk5/p35 may not be specific to tNhtt but may also affect the aggregation of other $\operatorname{poly}(\mathrm{Q})$ proteins. To address this question, we used atrophin-1, a protein product of the causative gene for DRPLA (dentatorubral and pallidoluysian atrophy) (Yamada et al., 2001), with 19Q and 81Q polyglutamine repeats. Inclusions of atrophin-1 with $81 \mathrm{Q}$ were decreased by cotransfection of Cdk5/ p35 (Fig. 2 D). These results suggest that Cdk5/p35 generally suppresses inclusion body formation of $\operatorname{poly}(\mathrm{Q})$ proteins indirectly through other cellular components.

\section{MTs and mhtt inclusions}

We suspected MTs as an intermediate component connecting Cdk5 activity and mhtt aggregation because MTs are a major target for Cdk5 (Niethammer et al., 2000) and are required for aggregate formation of mhtt (Muchowski et al., 2002; Taylor et al., 2003; Webb et al., 2004). To test this idea, we first assessed the requirement of MTs in the formation of mhtt inclusion bodies in COS-7 cells. When MTs were disassembled by nocodazole, no 
inclusions were formed (Fig. 3A), confirming previous results (Muchowski et al., 2002; Taylor et al., 2003; Webb et al., 2004). To test whether inclusions once formed can disintegrate, cells were treated with nocodazole for $6 \mathrm{~h}$ starting $18 \mathrm{~h}$ after transfection with $90 \mathrm{Q}$. Large inclusions were still observed, although smaller foci were not detected (Fig. 3A).

Next, we stabilized MTs with Taxol, which induces polymerization of randomly oriented noncentriolar MTs in the cytoplasm. Interestingly, many small inclusions of $50 \mathrm{Q}$ or $90 \mathrm{Q}$ were distributed around nucleus in Taxol-treated cells (Fig. $3 B$ ). We used tau as a neuronal protein to increase the stability of MTs. 16Q distributed diffusely in the whole cytoplasm whether or not tau was coexpressed. Smaller inclusions of $90 \mathrm{Q}$ were distributed in the cytoplasm and around the nucleus (Fig. 3C), as were observed in Taxoltreated cells. Whether the disruption of dynein motor function may alter the inclusions of tNhtt-poly(Q)-EGFP was examined by overexpression of dynamitin, a component of dynactin complex (Hirose et al., 2004). When $90 \mathrm{Q}$ was cotransfected with dynamitin, many small inclusions were observed only in the cytoplasm, particularly at the cell periphery (supplemental Fig. S2, available at www.jneurosci.org as supplemental material), consistent with previous results (Taylor et al., 2003). Together, these results indicate that intact MTs are required for the formation of inclusion bodies, and sizes and distribution of inclusion bodies are strongly dependent on MT organization.

\section{Disruption of the MT network by Cdk5/p35}

We then examined whether Cdk5/p35 affects MT organization in COS-7 cells. When Cdk5 and p35 were overexpressed, the radial MT networks observed in control cells disappeared and cytoplasmic tubulin staining was diffuse (Fig. 4A). To see the effect of Cdk5/p35 on MT stability biochemically, we fractionated cellular tubulin of COS-7 cells into polymeric and protomeric pools by mild treatment with MT-stabilizing buffer containing $0.5 \%$ Nonidet P-40, and quantified the amounts of tubulin in respective fractions by immunoblotting (see Materials and Methods). When COS-7 cells were treated with nocodazole, most of the tubulin was detected in the supernatant (Fig. $4 A$, Noc). In contrast, when COS-7 cells were treated with Taxol, almost all of the tubulin was recovered in the pellet fraction (Fig. 4A, Tax). When COS-7 cells transfected with Cdk5/p35 were fractionated similarly, polymeric tubulin decreased to $44 \%$ from $73 \%$ that was observed in control COS-7 cells transfected with empty vector, indicating that overexpression of Cdk5/p35 destabilizes MTs. These results agreed with our hypothesis that Cdk5/p35 suppresses mhtt aggregate formation through the disruption of MT networks. Next, we expressed tNhtt-poly(Q)-EGFP in COS-7 cells and observed MT networks. In addition to radial MTs, discrete tubulin staining was observed in the cytoplasm and at the cell periphery (Fig. $4 \mathrm{~B}$, arrows). We found that $16 \mathrm{Q}$ partly colo-
B

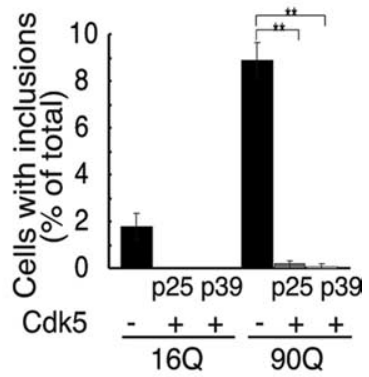

D

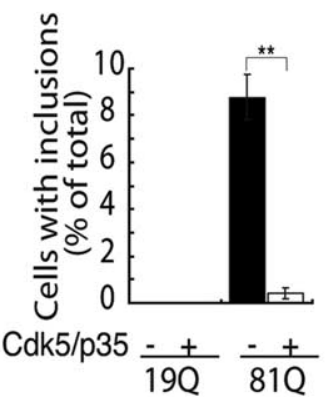

Figure 2. Requirement of Cdk5 kinase activity for the suppression of mhtt aggregate formation. $A$, tNhtt- 160,500, or 900 -

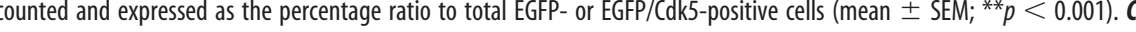
counted and expressed as the percentage ratio to total EGFP- or EGFP/Cdk5-positive cells (mean $\pm \mathrm{SEM}$; ${ }^{* *} p<0.001$ ). D Atrophin- 1 with 190 or 810 was cotransfected into COS-7 cells alone or with C $d k 5$ and p35 and cells containing EGFP inclusions were counted and expressed as the percentage ratio to total EGFP- or EGFP/Cdk5-positive cells (mean \pm SEM; ${ }^{* *} p<0.001$ ).

calized with MTs, as reported previously (Hoffner et al., 2002). The large inclusions of $50 \mathrm{Q}$ and $90 \mathrm{Q}$ were located in the perinuclear region, from which MTs emanate.

Using confocal microscopy, we visualized MTs and mhtt inclusions in COS-7 cells expressing Cdk5/p35. In the absence of Cdk5/p35, confocal images show large inclusions of 90Q in the cytoplasm (Fig. 4C, left panels) and sometimes in the nucleus (data not shown). MTs were excluded from these inclusions, and many small amorphous regions of tubulin staining were observed, in addition to randomly oriented short MTs (Fig. 4C, middle panel). In cells cotransfected with $90 \mathrm{Q}, \mathrm{Cdk} 5$, and p35, the staining of $90 \mathrm{Q}$ was diffuse in the cytoplasm. Unlike cells expressing Cdk5/p35 alone in which MTs were disassembled, some filamentous MT staining was observed in 90Q-transfected cells although the MTs were still disorganized (Fig. 4A,C).

$\mathrm{Cdk} 5 / \mathrm{p} 35$ is a multifunctional protein kinase that phosphorylates various proteins. To determine whether $\mathrm{Cdk} 5 / \mathrm{p} 35$ suppresses the formation of mhtt inclusion bodies through disruption of the MT network, COS-7 cells expressing 90Q, Cdk5, and p35 were treated with Taxol and inclusion formation was observed. The coexpression of Cdk5/p35 suppressed 90Q inclusion formation (Fig. $4 D$, left panel) as shown above. Taxol treatment induced the formation of inclusion bodies even in the presence of exogenous Cdk5/p35 expression. Small inclusions were scattered in the cytoplasm, as was observed in Taxol-treated and 90Qexpressing COS-7 cells (Fig. 3B). The number of cells bearing inclusions was twice as many as those of COS-7 cells expressing 90Q alone (Fig. $4 D$, right panel). Thus, the inhibitory action of 

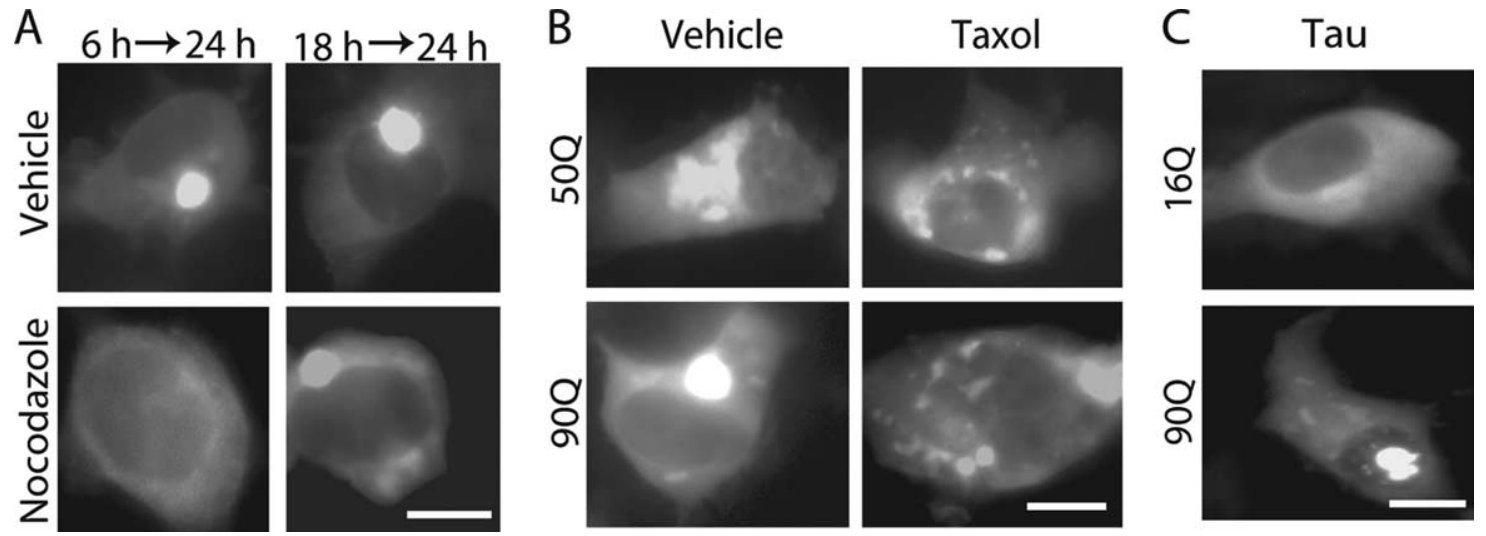

Figure 3. Inclusion formation is dependent on microtubule organization. A, COS-7 cells expressing tNhtt-90Q-EGFP were treated with $100 \mathrm{ng} / \mathrm{ml}$ nocodazole for $18 \mathrm{~h}$ starting $6 \mathrm{~h}$ after transfection or for $6 \mathrm{~h}$ starting $18 \mathrm{~h}$ after transfection. At the end of treatment, cells were analyzed for inclusion formation. Typical EGFP fluorescence images are shown. Cells containing EGFP inclusions were either 0 or $9.3 \pm 0.4 \%$ of the total EGFP-positive cells when treated with nocodazole or not, respectively. B, COS-7 cells expressing tNhtt-50Q or 90Q-EGFP were treated with $20 \mu \mathrm{m}$ Taxol for $18 \mathrm{~h}$ starting $6 \mathrm{~h}$ after transfection. At the end of treatment, cells were observed for inclusion formation. Cells containing EGFP inclusions were either 17.5 or $8.5 \%$ of the total EGFP-positive cells when treated with Taxol or not, respectively. C, tNhtt-160 or 90Q-EGFP was cotransfected into COS-7 cells with tau. Cells were analyzed for inclusion formation $24 \mathrm{~h}$ after transfection. Cells containing EGFP inclusions were either $11.7 \pm 0.7$ or $9.3 \pm 0.4 \%$ of the total 900 EGFP-positive cells when cotransfected with tau or not, respectively. Scale bars, $10 \mu \mathrm{m}$.
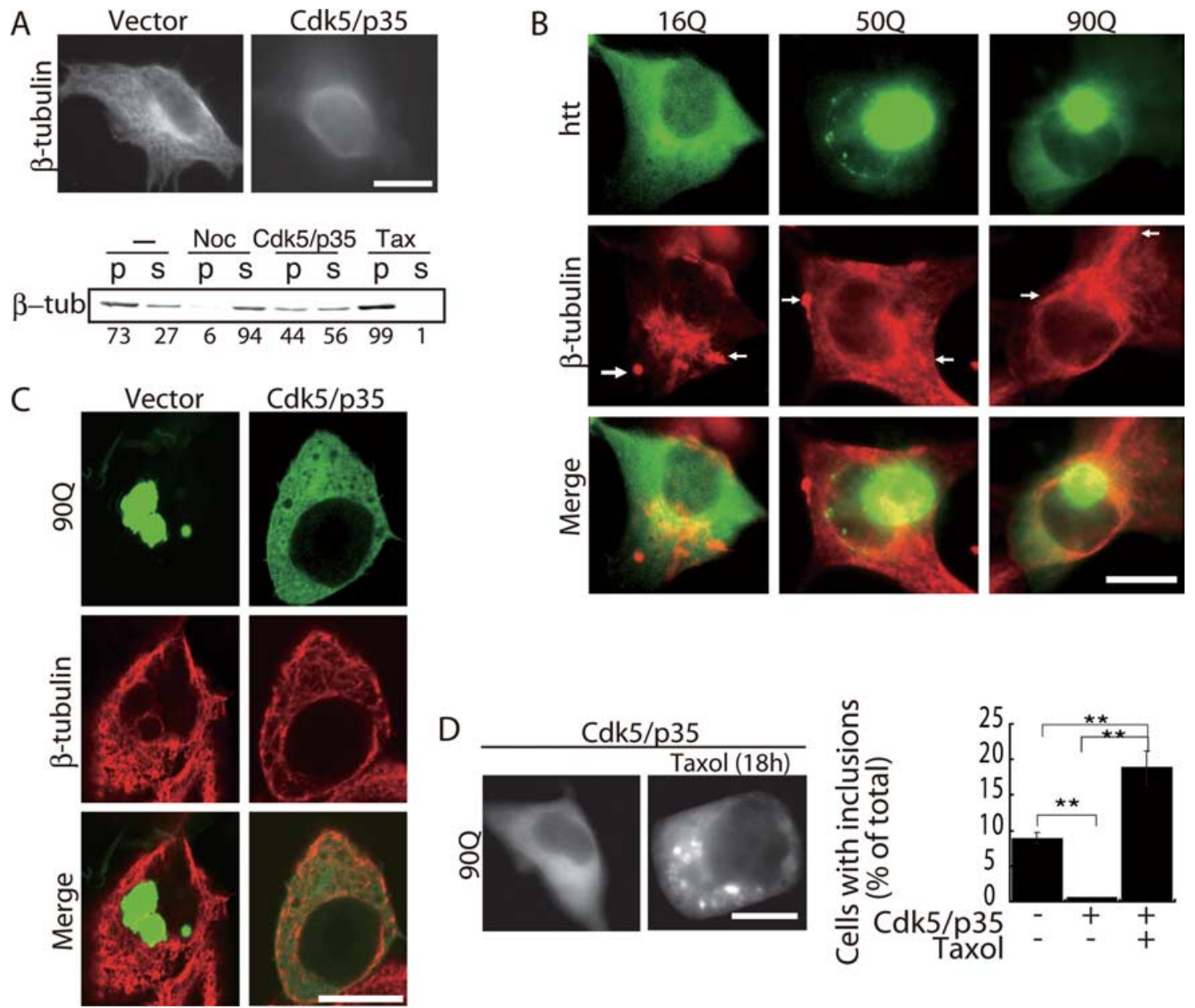

Figure 4. The MT network in COS-7 cells expressing (dk5, p35, and/or mhtt. $A$, MTs in COS-7 cells expressing Cdk5 and p35 were observed by immunofluorescence staining with anti- $\beta$-tubulin antibody $24 \mathrm{~h}$ after transfection (top). Bottom, Immunoblots showing the amount of $\beta$-tubulin in the pellet (p) and supernatant (s) in control cells ( - ), cells treated with nocodazole for $18 \mathrm{~h}$ (Noc), transfected with $\mathrm{Cdk5} / \mathrm{p} 35$ (Cdk5/p35), or treated with Taxol for $18 \mathrm{~h}$ (Tax). Polymeric and protomeric tubulin are expressed as the percentage ratio to total $\beta$-tubulin under the each lane. $\boldsymbol{B}$, MT distribution in COS-7 cells expressing tNhtt-16Q, 50Q, or 90Q-EGFP. MTs were stained with anti- $\beta$-tubulin antibody (red), and tNhtt-poly(Q)-EGFP was detected by EGFP fluorescence (green). The merged image is shown (bottom). The arrows indicate the discrete tubulin staining observed in the cytoplasm and at the cell periphery. C, Confocal microscopic images of tNhtt-90Q-EGFP (green; top), anti- $\beta$-tubulin staining (red; middle), and merged images (bottom) in COS-7 cells expressing Cdk5/p35 or not (vector). D, tNhtt-90Q-EGFP was transfected into COS-7 cells alone or with (dk5 and p35. Cells were treated with $20 \mu \mathrm{m}$ Taxol for $18 \mathrm{~h}$ starting $6 \mathrm{~h}$ after transfection. At the end of treatment, cells were observed for inclusions (left). Cells containing EGFP inclusions were counted and expressed as the percentage ratio to total EGFP-positive cells (right; mean \pm SEM; ${ }^{* *} p<0.001$ ). Scale bars, $10 \mu \mathrm{m}$. 
Cdk5/p35 on mhtt inclusion formation was canceled by the stabilization of MTs with Taxol.

\section{Regulation of inclusion formation by Cdk5/p35 in primary neurons}

We investigated whether we can regulate aggregate formation in neurons by manipulating Cdk5/p35 activity. First, we characterized the formation of inclusion bodies of mhtt in primary cultures of rat cortical neurons, which express Cdk5 and p35 endogeneously. When several concentrations of 166Q plasmids were introduced into neurons by a lipofection method, a number of neurons with inclusion bodies increased in parallel with the concentrations of plasmid used (Fig. 5A). To examine whether inclusion body formation is affected by MTs also in neurons, neurons transfected with 166Q were treated with Taxol. The percentage of neurons bearing inclusions increased significantly from $10.4 \pm 4.3$ to $33.4 \pm 0.5 \%(p<0.001)$ after treatment with Taxol, indicating that MTs play an important role in inclusion body formation in neurons.

We then introduced mhtt $(5 \mu \mathrm{g})$ into these neurons by calcium-phosphate transfection and observed mhtt inclusion formation. 90Q formed many small foci in the cytoplasm of primary neurons (Fig. $5 C$ ). When the kinase activity of Cdk5 was increased by infection of adenovirus encoding p35 (Fig. 5B, Flag-tagged p35), inclusion body formation was suppressed (Fig. 5C). Although we observed the suppression of inclusion body formation by increasing the activity of Cdk5 by p35 infection using Adenovirus vectors, we could not count sufficient numbers of neurons for statistical analysis.

We then asked whether aggregate formation is enhanced when Cdk 5 activity is decreased. We first used siRNA method to downregulate Cdk5 activity. Using Lipofectamine 2000 reagent, 90Q was cotransfected into primary neurons together with Cdk5 siRNA or control siRNA. When 90Q alone $(1 \mu \mathrm{g})$ was introduced into primary neurons by Lipofectamine 2000, we observed diffuse cytoplasmic distribution with stronger fluorescence in the perinuclear region in most neurons (Fig. 5E). When Cdk5 expression was reduced by RNA interference (Fig. 5D), neurons with discrete inclusion bodies of 90Q increased from $7.7 \pm 2.5$ to $18.2 \pm 7 \%(p<0.001)$. In addition, when $\mathrm{p} 35$ was reduced in primary cortical neurons by p35 siRNA, there was also an increase in the percentage of neurons with inclusions (data not shown).

To demonstrate the effect of Cdk5 on inclusion body formation in neurons more clearly, we next used primary neurons prepared from Cdk5-deficient mouse brains. Using Lipofectamine 2000 reagent, 166Q $(1 \mu \mathrm{g})$ was transfected into primary neurons of wild-type $\left(\mathrm{Cdk} 5^{+/+}\right)$and $\mathrm{Cdk} 5$ knock-out $\left(\mathrm{Cdk} 5^{-/-}\right)$mice (Fig. 6A). Inclusions were then observed at $24 \mathrm{~h}$ after transfection. There was a significant increase in the percentage of neurons control siRNA. Scale bars, $10 \mu \mathrm{m}$.
B
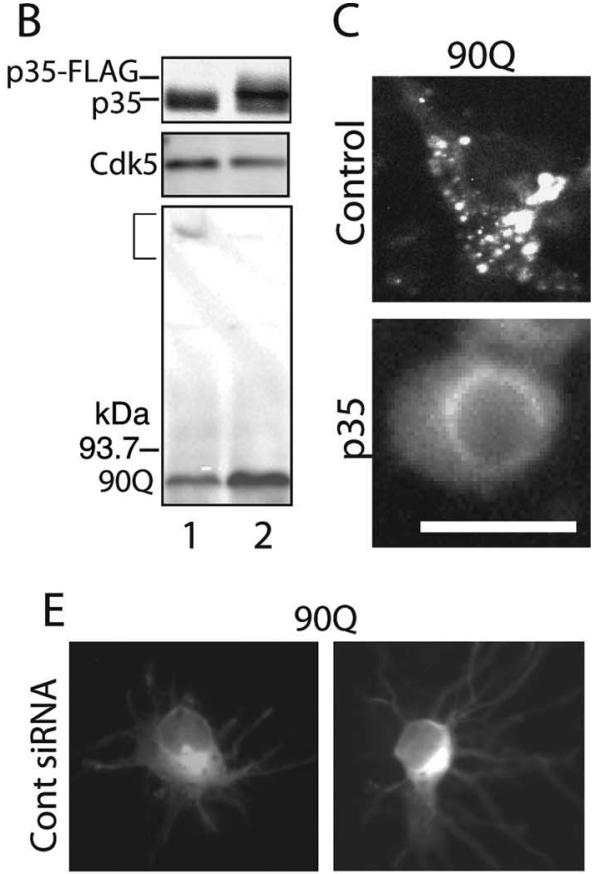

$90 \mathrm{Q}$
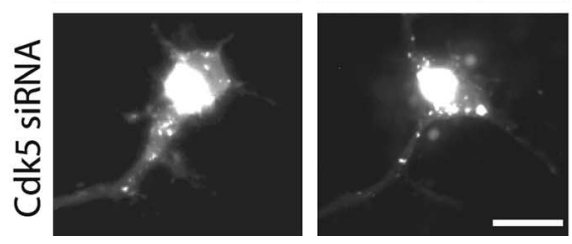

Figure 5. Modulation of mhtt inclusion formation by manipulating ( $\mathrm{kk} 5 / \mathrm{p} 35$ activity in primary cultured neurons. $\boldsymbol{A}$, Rat brain cortical neurons were transfected with different amounts of tNhtt-1660-EGFP plasmid DNA (in micrograms), and $18 \mathrm{~h}$ after

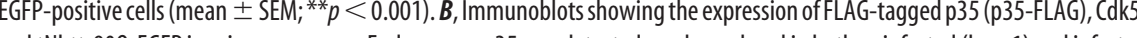
and tNhtt-900-EGFP in primary neurons. Endogenous $\mathrm{p} 35$ was detected as a lower band in both uninfected (lane 1) and infected 2) neurons. A bracket indicates SDS-insoluble aggregates in the stacking gel. C, Fluorescence images of EGFP in rat cortical control. $\boldsymbol{E}$, Fluorescence images of EGFP in rat cortical primary neurons transfected with tNhtt-900-EGFP and either Cdk5 siRNA or

with inclusions in $\mathrm{Cdk} 5^{-/-}$mice compared with wild-type mice (Fig. 6B). We also measured inclusion formation in Cdk5 $5^{+/-}$ neurons. Although there was no significant statistical difference from $\mathrm{Cdk} 5^{-1-}$ neurons, the ratio was always in between $\mathrm{Cdk} 5^{+/+}$and Cdk5 $5^{-1-}$ (Fig. 6B). These results demonstrate that Cdk5/p35 suppresses aggregate formation of mhtt in primary cultured neurons in a dose-dependent manner.

We compared the amount of MTs between Cdk $5^{-1-}$ and WT neurons by the same method used for COS-7 cells in Figure $4 \mathrm{~A}$. When we measured total tubulin, we found unexpectedly that tubulin in $\mathrm{Cdk} 5^{-1-}$ neurons was approximately two times $(203 \pm 28 \%$ when normalized by histone H3) larger than that in WT neurons (Fig. 6C). We then confirmed that the quantity of polymeric tubulin was higher in $\mathrm{Cdk} 5^{-1-}$ neurons than in WT neurons, although soluble tubulin was also increased in $\mathrm{Cdk} 5^{-1-}$ neurons (Fig. 6D).

\section{Discussion}

In this report, using an exon 1 fragment of htt containing the expanded poly(Q) (mhtt), 50Q, 90Q, and 166Q, we show that Cdk5/p35 suppresses mhtt aggregate formation in cell lines and in primary neuronal cultures. Although the mhtt we used was the $\mathrm{N}$-terminal exon 1 of htt without Cdk5/p35 phosphorylation 
A
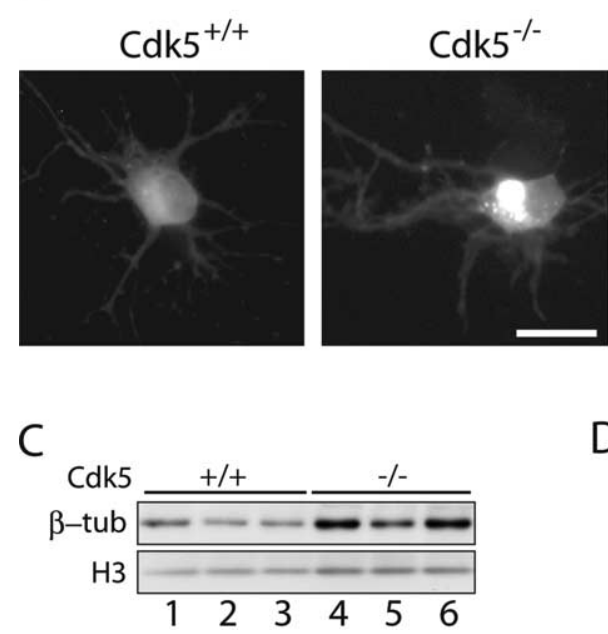

B

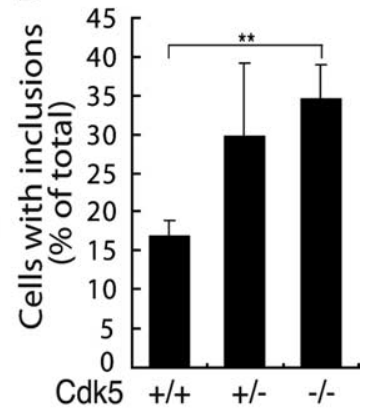

D

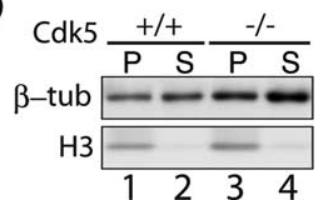

Figure 6. mhtt inclusion formation in primary cultured neurons of $C \mathrm{dk} 5^{-1-}$ mouse. $\boldsymbol{A}$, Fluorescence images of EGFP in $\mathrm{Cdk5}^{+/+}$or $\mathrm{Cdk5} 5^{-1-}$ mouse cortical primary neurons transfected with tNhtt-1660-EGFP. Scale bar, $10 \mu \mathrm{m}$. $\boldsymbol{B}$, Neurons containing EGFP inclusions were counted and expressed as the percentage ratio to total EGFP-positive cells (mean \pm SEM; ${ }^{* *} p<$ 0.001). C, Immunoblot showing the expression of $\beta$-tubulin in control and $C d k 5^{-1-}$ neurons. $\boldsymbol{D}$, Immunoblot showing the amount of $\beta$-tubulin in the MT polymers (P) and protomeric soluble pool $(S)$ in $C \mathrm{dk} 5^{-/-}$and control neurons.

tion sites. In fact, it was not phosphorylated by $\mathrm{Cdk} 5 / \mathrm{p} 35$. Nonetheless, the kinase activity of $\mathrm{Cdk} 5 / \mathrm{p} 35$ was needed to suppress aggregate formation in cultured cells and in primary neurons. The inhibitory effect of Cdk5/p35 was at least in part mediated by disruption of the MT network. Thus, Cdk5/p35 appears to be involved in mhtt pathogenesis both directly by phosphorylation of htt and indirectly through MTs at different levels; the toxicity of htt is exposed by DNA damage in a p53-dependent manner (Anne et al., 2007), the toxic N-terminal fragment is generated by caspase-dependent cleavage (Luo et al., 2005), and aggregates of expanded $\operatorname{poly}(\mathrm{Q})$ are formed in a MTdependent manner. Whereas the former two consequences are specific effects of Cdk5/p35 on htt protein, the last may be common to other poly $(\mathrm{Q})$-expanded proteins.

We suspected MTs as a likely candidate mediating the effects of Cdk5 because the MT network is a major target for Cdk5 and

sites, the kinase activity of Cdk5 was required for the suppression of aggregate formation. Having confirmed that intact MTs are required for the formation of mhtt inclusions and overexpression of Cdk5/p35 depolymerized MTs, we showed that the stabilization of MTs by Taxol eliminated the inhibitory action of Cdk5/ p35 on aggregate formation. The formation of mhtt inclusions was also modulated in primary neurons by manipulating Cdk5 activity or from mouse brains genetically manipulated. These results indicate that $\mathrm{Cdk} 5 / \mathrm{p} 35$ suppresses aggregate formation of mhtt through MT organization.

Recent studies suggest that inclusion bodies are nontoxic components resulting from sequestration or clearance of toxic species (Arrasate et al., 2004), although this question is not fully settled. The formation of inclusion bodies is a multistep process starting from mhtt monomers and ending with a large juxtanuclear deposit (Tanaka et al., 2003; Taylor et al., 2003; Nagai et al., 2007). We assayed the inhibitory activity of Cdk $5 / \mathrm{p} 35$ on mhtt inclusions in most of the experiments of this study, but we also observed that the formation of SDS-insoluble aggregates was inhibited by Cdk5/p35 as well. Thus, Cdk 5 appears to suppress the formation of mhtt inclusions at an early step of SDS-insoluble aggregate formation. Accordingly, we will use the term "aggregate" hereafter for the molecular species of mhtt, the formation of which Cdk5 affect.

Recently, it was shown that Cdk5 phosphorylates htt to alleviate the toxicity of mhtt (Luo et al., 2005; Anne et al., 2007). Phosphorylation of htt at Ser434 by Cdk5 attenuates aggregate formation by preventing the caspase-dependent cleavage of htt (Luo et al., 2005). When striatal neurons suffer from DNA damage, nuclear htt is phosphorylated at Ser1181 and Ser1201 by Cdk5 (Anne et al., 2007). Phosphorylation at Ser1181 and Ser1201 not only protects from poly $(\mathrm{Q})$-induced neuronal death in p53-dependent manner but also protects against poly(Q)induced toxicity. However, the inhibitory effect of Cdk5 on aggregate formation we observed here is distinct from those described in the above two studies. The N-terminal fragment of htt exon 1 we used here is shorter than those used by Luo et al. (2005) and Anne et al. (2007) and does not contain Cdk5 phosphoryla- is required for inclusion formation of mhtt and other poly $(\mathrm{Q})$ proteins (Muchowski et al., 2002; Taylor et al., 2003; Webb et al., 2004). From four independent experiments using nocodazole, Taxol, tau, and dynamitin, we confirmed that MTs are a critical component affecting both the size and cellular location of mhtt inclusion bodies. MTs in COS-7 cells disassembled and tubulin staining became diffuse in the cytoplasm when $\mathrm{Cdk} 5 / \mathrm{p} 35$ was coexpressed with tNhtt-poly(Q)-EGFP, as occurred with nocodazole treatment. Although it was recently reported that p35 bound and stabilized MTs in vitro (Hou et al., 2007), MT stabilization was not observed in our cultured cells. Our results are consistent with previous reports demonstrating the requirement of MTs in cytoplasmic aggregate formation (Johnston et al., 1998; Iwata et al., 2005). The disruption of MTs in a particular cellular region may suppress the accumulation of mhtt below concentrations required to initiate inclusion body formation. The importance of local concentrations of mhtt for an initial step of aggregate formation has been demonstrated previously (Hazeki et al., 1999). Although the cancellation of the inhibitory effect of Cdk5/ p35 by Taxol implicates MTs as a major downstream target of Cdk5/p35, the interpretation should be cautious until the identification of the target protein. Multiple Cdk5 target proteins affect MT functions, such as motor-associated proteins for MTdependent trafficking and microtubule-associated proteins (MAPs) for MT stabilization (Patrick et al., 1999; Niethammer et al., 2000; Sakaue et al., 2005; Caviston et al., 2007). Cdk5/p35 may inactivate the stabilizing activity of MAPs or may activate destabilizing factors by phosphorylation. It is well known that phosphorylation of tau, a major neuronal MAP, by Cdk 5 decreases its MT stabilizing ability (Wada et al. 1998). The identification of the target substrate for $\mathrm{Cdk} 5 / \mathrm{p} 35$ would be a critical question to be answered.

A remaining question is whether the inhibitory action of Cdk5/p35 on aggregate formation is protective. Aggregates we observed here are mostly cytoplasmic, whereas nuclear inclusions are observed in HD brains (DiFiglia et al., 1997). Perinuclear aggregates are suggested to be protective. Nocodazole treatment increases the ratio of cell death despite decreased mhtt inclusions 
(Muchowski et al., 2002; Taylor et al., 2003; Webb et al., 2004). These results were interpreted to indicate that suppression of inclusion body formation increases the toxic species that are otherwise sequestrated into nontoxic inclusions (Taylor et al., 2003). According to this scenario, Cdk5/p35 might enhance the toxicity of mhtt by inhibiting the clearance of the toxic component. However, unlike nocodazole treatment in HEK293 cells expressing a poly(Q)-androgen receptor (AR-112Q) after which SDSinsoluble aggregates persist (Taylor et al., 2003), the SDSinsoluble aggregates of mhtt disappeared with coexpression of Cdk5/p35. Thus, Cdk5/p35 appears to inhibit the formation of SDS-insoluble oligomers. If the SDS-insoluble oligomers are toxic species, Cdk5/p35 would work as a protective factor.

mhtt aggregate formation in primary neurons was regulated by Cdk 5 activity. When Cdk 5 activity was increased by p 35 expression, aggregate formation was suppressed. In contrast, when Cdk5 activity was downregulated by gene knock-out or siRNA technique, aggregate formation was enhanced. The balance between Cdk5 activity and mhtt protein concentration may be critical. Aggregate formation should also be increased in neurons because the expression levels of MTs are high. Cdk5 kinase activity is reduced in HD mouse models (Luo et al., 2005), and downregulation of Cdk5 is observed in the brains of HD patients (Tam et al., 2006). As a result of reduced Cdk5 activity, MTs may be overstabilized, or transport on MTs may be biased in a particular direction, resulting in higher local concentrations of mhtt in neurons of HD patients. In contrast, it is well known that abnormal activation of Cdk5 in neurons causes hyperphosphorylation of tau, possibly leading to aggregation of tau and then to neuronal cell death in Alzheimer's disease (Patrick et al., 1999; Noble et al., 2003; Cruz et al., 2006). Thus, proper regulation of Cdk5 kinase activity may be necessary for neuronal survival. Moreover, manipulating Cdk5 kinase activity in neurons may provide the possibility of regulating development of neurodegenerative diseases such as HD and Alzheimer's disease.

\section{References}

Anne SL, Saudou F, Humbert S (2007) Phosphorylation of huntingtin by cyclin-dependent kinase 5 is induced by DNA damage and regulates wildtype and mutant huntingtin toxicity in neurons. J Neurosci 27:7318-7328.

Arrasate M, Mitra S, Schweitzer ES, Segal MR, Finkbeiner S (2004) Inclusion body formation reduces levels of mutant huntingtin and the risk of neuronal death. Nature 431:805-810.

Bennett EJ, Shaler TA, Woodman B, Ryu KY, Zaitseva TS, Becker CH, Bates GP, Schulman H, Kopito RR (2007) Global changes to the ubiquitin system in Huntington's disease. Nature 448:704-708.

Cattaneo E, Zuccato C, Tartari M (2005) Normal huntingtin function: an alternative approach to Huntington's disease. Nat Rev Neurosci 6:919-930.

Caviston JP, Ross JL, Antony SM, Tokito M, Holzbaur EL (2007) Huntingtin facilitates dynein/dynactin-mediated vesicle transport. Proc Natl Acad Sci U S A 104:10045-10050.

Cheung ZH, Ip NY (2007) The roles of cyclin-dependent kinase 5 in dendrite and synapse development. Biotechnol J 2:949-957.

Cruz JC, Kim D, Moy LY, Dobbin MM, Sun X, Bronson RT, Tsai LH (2006) p25/cyclin-dependent kinase 5 induces production and intraneuronal accumulation of amyloid beta in vivo. J Neurosci 26:10536-10541.

Davies SW, Turmaine M, Cozens BA, DiFiglia M, Sharp AH, Ross CA, Scherzinger E, Wanker EE, Mangiarini L, Bates GP (1997) Formation of neuronal intranuclear inclusions underlies the neurological dysfunction in mice transgenic for the HD mutation. Cell 90:537-548.

Dhavan R, Tsai LH (2001) A decade of CDK5. Nat Rev Mol Cell Biol 2:749-759.

DiFiglia M, Sapp E, Chase KO, Davies SW, Bates GP, Vonsattel JP, Aronin N (1997) Aggregation of huntingtin in neuronal intranuclear inclusions and dystrophic neurites in brain. Science 277:1990-1993.
Gauthier LR, Charrin BC, Borrell-Pagès M, Dompierre JP, Rangone H, Cordelières FP, De Mey J, MacDonald ME, Lessmann V, Humbert S, Saudou F (2004) Huntingtin controls neurotrophic support and survival of neurons by enhancing BDNF vesicular transport along microtubules. Cell 118:127-138.

Harjes P, Wanker EE (2003) The hunt for huntingtin function: interaction partners tell many different stories. Trends Biochem Sci 28:425-433.

Hazeki N, Nakamura K, Goto J, Kanazawa I (1999) Rapid aggregate formation of the huntingtin $\mathrm{N}$-terminal fragment carrying an expanded polyglutamine tract. Biochem Biophys Res Commun 256:361-366.

Hirose H, Arasaki K, Dohmae N, Takio K, Hatsuzawa K, Nagahama M, Tani K, Yamamoto A, Tohyama M, Tagaya M (2004) Implication of ZW10 in membrane trafficking between the endoplasmic reticulum and Golgi. EMBO J 23:1267-1278.

Hisanaga S, Saito T (2003) The regulation of cyclin-dependent kinase 5 activity through the metabolism of p 35 or p 39 Cdk5 activator. Neurosignals 12:221-229.

Hoffner G, Kahlem P, Djian P (2002) Perinuclear localization of huntingtin as a consequence of its binding to microtubules through an interaction with $\beta$-tubulin: relevance to Huntington's disease. J Cell Sci 115:941-948.

Hou Z, Li Q, He L, Lim HY, Fu X, Cheung NS, Qi DX, Qi RZ (2007) Microtubule association of the neuronal p35 activator of Cdk5. J Biol Chem 282:18666-18670.

Iwata A, Riley BE, Johnston JA, Kopito RR (2005) HDAC6 and microtubules are required for autophagic degradation of aggregated huntingtin. J Biol Chem 280:40282-40292.

Johnston JA, Ward CL, Kopito RR (1998) Aggresomes: a cellular response to misfolded proteins. J Cell Biol 143:1883-1898.

Kazantsev A, Walker HA, Slepko N, Bear JE, Preisinger E, Steffan JS, Zhu YZ, Gertler FB, Housman DE, Marsh JL, Thompson LM (2002) A bivalent Huntingtin binding peptide suppresses polyglutamine aggregation and pathogenesis in Drosophila. Nat Genet 30:367-376.

Li SH, Li XJ (2004) Huntingtin-protein interactions and the pathogenesis of Huntington's disease. Trends Genet 20:146-154.

Luo S, Vacher C, Davies JE, Rubinsztein DC (2005) Cdk5 phosphorylation of huntingtin reduces its cleavage by caspases: implications for mutant huntingtin toxicity. J Cell Biol 169:647-656.

Muchowski PJ, Ning K, D'Souza-Schorey C, Fields S (2002) Requirement of an intact microtubule cytoskeleton for aggregation and inclusion body formation by a mutant huntingtin fragment. Proc Natl Acad Sci U S A 99:727-732.

Nagai Y, Inui T, Popiel HA, Fujikake N, Hasegawa K, Urade Y, Goto Y, Naiki $\mathrm{H}$, Toda $\mathrm{T}$ (2007) A toxic monomeric conformer of the polyglutamine protein. Nat Struct Mol Biol 14:332-340.

Nguyen HL, Gruber D, Bulinski JC (1999) Microtubule-associated protein 4 (MAP4) regulates assembly, protomer-polymer partitioning and synthesis of tubulin in cultured cells. J Cell Sci 112:1813-1824.

Niethammer M, Smith DS, Ayala R, Peng J, Ko J, Lee MS, Morabito M, Tsai LH (2000) NUDEL is a novel Cdk5 substrate that associates with LIS1 and cytoplasmic dynein. Neuron 28:697-711.

Nikolic M, Dudek H, Kwon YT, Ramos YF, Tsai LH (1996) The cdk5/p35 kinase is essential for neurite outgrowth during neuronal differentiation. Genes Dev 10:816-825.

Noble W, Olm V, Takata K, Casey E, Mary O, Meyerson J, Gaynor K, LaFrancois J, Wang L, Kondo T, Davies P, Burns M, Veeranna, Nixon R, Dickson D, Matsuoka Y, Ahlijanian M, Lau LF, Duff K (2003) Cdk5 is a key factor in tau aggregation and tangle formation in vivo. Neuron 38:555-565.

Ohshima T, Ward JM, Huh CG, Longenecker G, Veeranna, Pant HC, Brady RO, Martin LJ, Kulkarni AB (1996) Targeted disruption of the cyclindependent kinase 5 gene results in abnormal corticogenesis, neuronal pathology and perinatal death. Proc Natl Acad Sci U S A 93:11173-11178.

Ohshima T, Suzuki H, Morimura T, Ogawa M, Mikoshiba K (2007) Modulation of Reelin signaling by Cyclin-dependent kinase 5 . Brain Res 1140:84-95.

Onodera O, Burke JR, Miller SE, Hester S, Tsuji S, Roses AD, Strittmatter WJ (1997) Oligomerization of expanded-polyglutamine domain fluorescent fusion proteins in cultured mammalian cells. Biochem Biophys Res Commun 238:599-605.

Patrick GN, Zukerberg L, Nikolic M, de la Monte S, Dikkes P, Tsai LH (1999) Conversion of p35 to p25 deregulates Cdk5 activity and promotes neurodegeneration. Nature 402:615-622.

Saito T, Onuki R, Fujita Y, Kusakawa G, Ishiguro K, Bibb JA, Kishimoto T, 
Hisanaga S (2003) Developmental regulation of the proteolysis of the p35 cyclin-dependent kinase 5 activator by phosphorylation. J Neurosci 23:1189-1197.

Sakaue F, Saito T, Sato Y, Asada A, Ishiguro K, Hasegawa M, Hisanaga S (2005) Phosphorylation of FTDP-17 mutant tau by cyclin-dependent kinase 5 complexed with p35, p25, or p39. J Biol Chem 280:31522-31529.

Sánchez I, Mahlke C, Yuan J (2003) Pivotal role of oligomerization in expanded polyglutamine neurodegenerative disorders. Nature 421:373-379.

Scherzinger E, Lurz R, Turmaine M, Mangiarini L, Hollenbach B, Hasenbank R, Bates GP, Davies SW, Lehrach H, Wanker EE (1997) Huntingtinencoded polyglutamine expansions form amyloid-like protein aggregates in vitro and in vivo. Cell 90:549-558.

Shelton SB, Johnson GV (2004) Cyclin-dependent kinase-5 in neurodegeneration. J Neurochem 88:1313-1326.

Tam S, Geller R, Spiess C, Frydman J (2006) The chaperonin TRiC controls polyglutamine aggregation and toxicity through subunit-specific interactions. Nat Cell Biol 8:1155-1162.

Tanaka M, Machida Y, Nishikawa Y, Akagi T, Hashikawa T, Fujisawa T, Nukina N (2003) Expansion of polyglutamine induces the formation of quasi-aggregate in the early stage of protein fibrillization. J Biol Chem 278:34717-34724.

Taylor JP, Tanaka F, Robitschek J, Sandoval CM, Taye A, Markovic-Plese S, Fischbeck KH (2003) Aggresomes protect cells by enhancing the degradation of toxic polyglutamine-containing protein. Hum Mol Genet 12:749-757.
Tobin AJ, Signer ER (2000) Huntington's disease: the challenge for cell biologists. Trends Cell Biol 10:531-536.

Wada Y, Ishiguro K, Itoh TJ, Uchida T, Hotani H, Saito T, Kishimoto T, Hisanaga S (1998) Microtubule-stimulated phosphorylation of tau at Ser202 and Thr205 by cdk 5 decreases its microtubule nucleation activity. J Biochem (Tokyo) 124:738-746.

Wang GH, Mitsui K, Kotliarova S, Yamashita A, Nagao Y, Tokuhiro S, Iwatsubo T, Kanazawa I, Nukina N (1999) Caspase activation during apoptotic cell death induced by expanded polyglutamine in N2a cells. Neuroreport 10:2435-2438.

Webb JL, Ravikumar B, Rubinsztein DC (2004) Microtubule disruption inhibits autophagosome-lysosome fusion: implications for studying the roles of aggresomes in polyglutamine diseases. Int J Biochem Cell Biol 36:2541-2550.

Wyttenbach A, Carmichael J, Swartz J, Furlong RA, Narain Y, Rankin J, Rubinsztein DC (2000) Effects of heat shock, heat shock protein 40 (HDJ-2), and proteasome inhibition on protein aggregation in cellular models of Huntington's disease. Proc Natl Acad Sci U S A 97:2898-2903.

Yamada M, Wood JD, Shimohata T, Hayashi S, Tsuji S, Ross CA, Takahashi H (2001) Widespread occurrence of intranuclear atrophin-1 accumulation in the central nervous system neurons of patients with dentatorubralpallidoluysian atrophy. Ann Neurol 49:14-23.

Yamashita N, Morita A, Uchida Y, Nakamura F, Usui H, Ohshima T, Taniguchi M, Honnorat J, Thomasset N, Takei K, Takahashi T, Kolattukudy P, Goshima Y (2007) Regulation of spine development by semaphorin3A through cyclin-dependent kinase 5 phosphorylation of collapsin response mediator protein 1. J Neurosci 27:12546-12554. 